\section{Relación entre las modalidades de ingreso y el rendimiento académico de los estudiantes de estomatología de las cohortes 2008-2009 en una Universidad Privada}

Flores-Mori $M^{1}$. Relación entre las modalidades de ingreso y el rendimiento académico de los estudiantes de estomatología de las cohortes 2008-2009 en una Universidad Privada. Rev Estomatol Herediana. 2012 Oct-Dic;22(4):210-5.

\section{RESUMEN}

Objetivos: Determinar la relación entre las modalidades de ingreso y el rendimiento académico universitario de los estudiantes de estomatología de las cohortes 2008-2009 de la Universidad Peruana Cayetano Heredia (UPCH). Material y métodos: Diseño de corte descriptivo, observacional, longitudinal y retrospectivo, se realizó en la población total de estudiantes de la Facultad de Estomatología Roberto Beltrán de la UPCH que ingresaron en los años 2008 y 2009 que cursaron el primer y segundo año académico. En el 2008 ingresaron 118 y en el 2009 fueron 110. Resultados: Respecto a las modalidades de ingreso se observa que el mayor número de estudiantes ingresaron por Factor Excelencia $(\mathrm{N}=135,59 \%)$, seguido de Examen de admisión $(\mathrm{N}=52,22 \%)$ y Centro Preuniversitario-CEPREU ( $\mathrm{N}=38,16 \%)$, respecto al rendimiento académico universitario según género se encontró que el mayor número de estudiantes fueron de sexo femenino $(\mathrm{N}=159,70 \%)$ seguido del sexo masculino $(\mathrm{N}=69, \quad 30 \%)$, no se encontró diferencias estadísticamente significativas respecto al rendimiento académico universitario según sexo. Conclusiones: Respecto al estudio de la relación del rendimiento académico universitario con las modalidades de ingreso se encontró que los estudiantes obtuvieron promedios similares en el rendimiento académico universitario al margen de la modalidad de ingreso, en términos de promedio ponderado anual, la relación no fue estadísticamente significativa $(\mathrm{P}>0,05)$. Las modalidades de admisión parecen no ser un buen predictor del rendimiento académico; salvo el rendimiento escolar previo, una vez que el estudiante ingresa a la universidad se disipan algunas variables que los caracterizan durante la etapa escolar.

Palabras clave: EDUCACIÓN SUPERIOR, ESTUDIANTES DE ODONTOLOGÍA, EDUCACIÓN PRE ODONTOLÓGICA.

Relationship between different admissions criteria and academic performance of the career of dentistry in a Private University.

\section{ABSTRACT}

Objectives: The increasing number of students attending university raises the interest to know the factors able to predict academic performance in the university studies. This paper studies the association of the academic university performance in the first two years of studies of the career of dentistry of the Universidad Peruana Cayetano Heredia to different admission criteria to the University. Material and methods: The study was descriptive and correlational research in a population of 228 students of both sexes from the first and second year of studies of the cohorts 2008 and 2009. Results: The research shows no association of university academic performance with sex and different admission criteria on school institution. Dental schools should assess the validity of their selection systems. Conclusions: Further longitudinal, national work is nevertheless required to determine the extent to which high school performance, sociodemographic and educational variables predict academic performance on undergraduate programs.

\section{Mirza Flores-Mori ${ }^{1}$}

Cirujano dentista, Magister en Educación, Docente del Departamento Académico de Estomatología del Niño y del Adolescente, Facultad de Estomatología Roberto Beltrán, Universidad Peruana Cayetano Heredia. Lima, Perú.

\section{Correspondencia:}

Mirza Flores Mori

Av. Honorio Delgado № 430, San Martín de Porres.

Lima, Perú.

E-mail: mirza.flores@upch.pe

Keywords: EDUCATION, HIGHER, STUDENTS, DENTAL, PREDENTAL.

\section{Introducción}

El análisis del problema del sistema de selección y admisión de postulantes a la universidad es una materia que necesariamente con- grega criterios técnicos, políticas sociales y efectos personales. Ello la hace un área de gran relevancia tanto para los actores individuales, como para los sociales, entendién- dose por estos últimos a las instituciones universitarias, el mundo laboral y la sociedad como tal pues generarán consecuencias sobre tales dimensiones (1). 
Para asegurar la calidad de la educación superior, se debe partir de la selección de los estudiantes considerando los factores que influyen sobre el rendimiento académico y que repercuten en la deserción estudiantil sobre todo durante la transición del colegio a la universidad. La universidad sólo debe admitir como estudiantes a aquellas personas que tengan un legítimo y autentico interés por el conocimiento como factor necesario para el avance social (2).

El rendimiento académico es definido por Chadwick como la expresión de capacidades y de características psicológicas del estudiante desarrolladas y actualizadas a través del proceso de enseñanzaaprendizaje que le posibilita obtener un nivel de funcionamiento $\mathrm{y}$ logros académicos a lo largo de un periodo o semestre que se sintetiza en un calificativo final (cuantitativo en la mayoría de los casos) evaluador del nivel alcanzado (3). Este es un problema que trasciende los niveles y épocas, y es una de las principales preocupaciones para quienes están comprometidos con la calidad educativa, es considerado un componente clave para determinar si una institución está alcanzando sus objetivos educativos (4).

Ramallo y Sigal proponen una tipología para discriminar los diferentes niveles de intensidad o exigencia de los sistemas de selección a la universidad, mostrando cuán alto es el grado de dificultad para los postulantes el vincularse al ámbito universitario (5) (Tabla 1).

En Perú no se cuenta con un examen de admisión nacional que permita medir la eficacia de la educación secundaria. El sentido de los procesos de selección a la universidad es la selección de los mejores postulantes, hasta cubrir un determinado número de vacantes. Sin embargo no consideran una nota mínima aprobatoria por tanto, no garantizan una adecuada selección de postulantes. El grado de selectividad en el ingreso a la universidad toma como dato confiable la proporción entre los postulantes e ingresantes (Gráfico 1). Encontrándose una selectividad marcadamente más alta en las universidades públicas, siendo la tasa hacia el incremento constante contrariamente a la situación de las universidades privadas alcanzando en algunas instituciones el nivel cero o paradójicamente negativo. Algunas instituciones incluyen en las modalidades de selección las notas escolares $\mathrm{y}$ en muy pocos casos se incluye test vocacionales o de capacidades especiales. La Tabla 2 presenta la relación de universidades privadas que ofrecen dentro de su sistema de selección, modalidades que toman en consideración el rendimiento escolar previo (6).

Numerosos estudios respecto a la validez predictiva de los diferentes sistemas de selección y su correlación con el rendimiento académico universitario han mostrado una correlación positiva, dándole gran valor predictivo del futuro rendimiento académico $(1,7,10)$. El estudio realizado por Poussaint en la Universidad de Harvard demuestra que los resultados de las pruebas de admisión pueden sugerir éxito docente en el ciclo básico, no así para el desempeño clínico (8).

El presente estudio permitió evaluar la relación entre las modalidades de ingreso y el rendimiento académico universitario en la Facultad de Estomatología de la Universidad Peruana Cayetano Heredia (UPCH), específicamente durante los dos primeros años. Esto permitirá analizar la efectividad de los sistemas de admisión, afianzar o reorientar los sistemas de admisión existentes e implementar otros basados en instrumentos válidos y confiables a fin de seleccionar postulantes capaces, que permitan a la institución cumplir con el perfil profesional que postula, así como evaluar la calidad de la docencia en la institución.

\section{Material y Métodos}

Diseño de tipo descriptivo, observacional, longitudinal y retrospectivo. Se estudió la asociación entre las modalidades de ingreso y el rendimiento académico de los estudiantes de las cohortes 2008 y 2009.

El estudio se realizó en la población total de estudiantes que ingresaron en los años 2008 y 2009 que cursaron el primer y segundo año académico. El 2008 ingresaron 118 y en el 2009 fueron 110 estudiantes.

La base de datos fue tomada de la Secretaria Académica de la Facultad de Estomatología que consigna los expedientes de los postulantes a la facultad y el record académico por año.

\section{Criterios de inclusión:}

1. Estudiantes que ingresaron por las diferentes modalidades de ingreso a la Facultad.

2. Estudiantes que cursaron todas las asignaturas del primer y segundo año académico correspondiente a su cohorte.

El rendimiento académico universitario se consideró como variable dependiente, y la modalidad de ingreso como variable interviniente. Se consideró el sexo como covariable. El rendimiento académico universitario se calculó a partir de las calificaciones obtenidas en las 
asignaturas cursadas, expresado por la sumatoria de los productos de las calificaciones obtenidas por el creditaje respectivo de la asignatura estudiada, dividido entre el creditaje total anual, durante los dos primeros años de formación universitaria.

\section{Sistema de admisión:}

Modalidades por las cuales el estudiante ingresó a la Facultad de Estomatología:

1. Centro de Estudios Pre-universitarios (CEPREU)

2. Factor Excelencia: Los que en el $3^{\circ}, 4^{\circ}$ y $5^{\circ}$ de secundaria obtuvieron un promedio de notas de 14 o más en cada año de estudios.

3. Examen General de Admisión.

4. Exoneración del procedimiento ordinario de admisión.

a. Titulados o Graduados en la UPCH en otros centros educativos de nivel superior del país o de universidades extranjeras.

b. Primeros o segundos puestos de su promoción en centros educativos de nivel secundario del país.

5. Los diplomados del Bachillerato Internacional

6. Admisión Extraordinaria.

a. Los cónyuges e hijos de diplomáticos y funcionarios internacionales extranjeros acreditados en el país.

b. Los cónyuges e hijos de diplomáticos peruanos que retornan al país.

c. Los deportistas calificados.

d. Las personas calificadas por su formación en el trabajo.

7. Traslado

\section{Plan de análisis}

Los datos obtenidos fueron ta- bulados y ordenados en orden decreciente del máximo puntaje de rendimiento académico hasta el mínimo.

Se tomó en cuenta un valor de $\mathrm{p}<$ 0,05 y el intervalo de confianza del 95\% como el nivel de significancia estadística. A los datos obtenidos se aplicó el paquete estadístico SPSS.

Para el análisis estadístico se emplearon los siguientes estadígrafos y pruebas estadísticas:

Media Aritmética, Desviación Estándar, t de Student y Análisis de Varianza (ANOVA).

El protocolo de investigación fue revisado y aprobado por el Comité de Etica de la UPCH.

\section{Resultados}

En lo que se refiere a la modalidad de ingreso, se observa que el mayor porcentaje de ingreso fue

Porcentaje de postulantes que ingresaron $1987,1991,1994$ y 2004

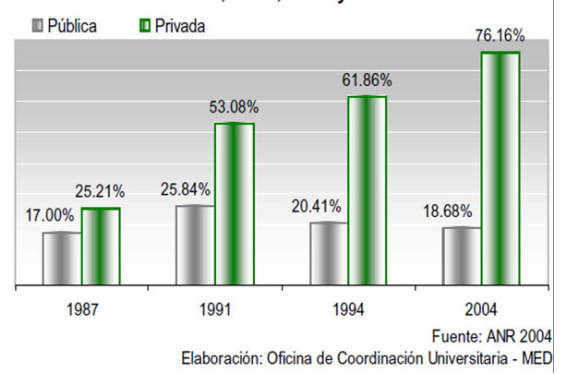

Gráfico 1. Porcentaje de postulantes que ingresaron a la universidad entre los años 1987 y 2004

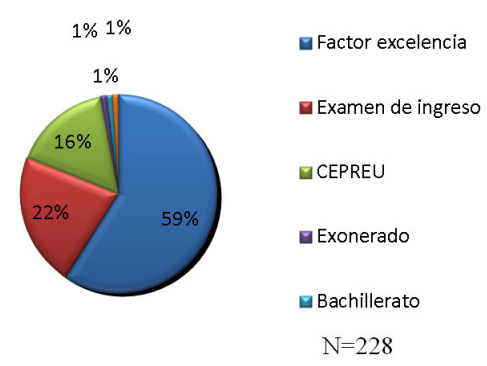

Gráfico 2. Distribución de los estudiantes según modalidad de ingreso

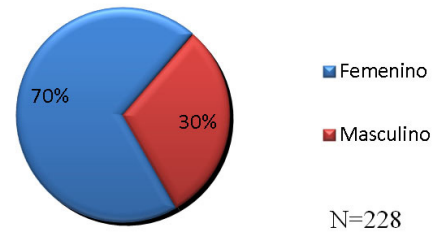

Gráfico 3. Distribución de los estudiantes según genero.

por factor de excelencia $(59,2 \%)$, seguido del examen de ingreso $(22,8 \%)$ y CEPREU $(16,7 \%)$ (Gráfico 2).

El mayor porcentaje de los estudiantes fue del sexo femenino $(69,7 \%)$ seguido del sexo masculino $(30,3 \%)$ (Gráfico 3).

El rendimiento académico en la cohorte 2008 fue 13,53 y en la cohorte 2009 fue 13,56 no encontrándose diferencias estadísticamente significativas (Tabla 3).

En lo que respecta al rendimiento académico según género, el rendimiento de los alumnos del género masculino fue de 16,76 y del femenino fue de 13,06 no se encontró diferencias estadísticamente significativas (Tabla 4).

Las medias del rendimiento académico universitario son similares según las modalidades de ingreso (Tabla 5).

\section{Discusión}

Las estimaciones permiten demostrar que respecto al estudio de la relación entre modalidades de ingreso y el rendimiento académico universitario no pudo ser comprobada, encontrándose que los estudiantes obtuvieron promedios similares en el rendimiento académico universitario al margen de la modalidad de ingreso, en términos de promedio ponderado anual la re- 
Tabla 1. Sistemas de admisión según nivel de intensidad

\begin{tabular}{ccl}
\hline Sistema de admisión & $\begin{array}{c}\text { Nivel de } \\
\text { intensidad }\end{array}$ & Descripción \\
\hline $\begin{array}{c}\text { Examen eliminatorio } \\
\text { con cupo }\end{array}$ & Alta & $\begin{array}{l}\text { Comprende evaluaciones diagnósticas, exámenes teóricos y } \\
\text { prácticos, el puntaje determina el cupo a la carrera selec- } \\
\text { cionada. }\end{array}$ \\
$\begin{array}{c}\text { Examen eliminatorio } \\
\text { sin cupo }\end{array}$ & Media & $\begin{array}{l}\text { Condicionado a la aprobación de un examen, sin establecer } \\
\text { el orden de mérito. } \\
\text { Examen no } \\
\text { eliminatorio }\end{array}$ \\
$\begin{array}{c}\text { Directo con o } \\
\text { sin instancia de } \\
\text { ambientación }\end{array}$ & Baja & $\begin{array}{l}\text { nósticas y exámenes, previos a ser considerado alumno } \\
\text { (carácter clasificatorio). }\end{array}$ \\
\hline
\end{tabular}

Fuente: Ramallo y Sigal (5)

Tabla 2. Universidades peruanas que ofrecen modalidades de ingreso tomando como referencia las notas obtenidas durante la etapa secundaria.
Institución Educativa

Universidad Peruana Cayetano Heredia

Pontifica Universidad Católica del Perú

Universidad del Pacífico

Universidad Peruana de Ciencias Aplicadas

Universidad Femenina del sagrado Corazón Universidad ESAN

Universidad de Lima

\section{Modalidad de ingreso}

\section{Factor Excelencia}

ITS (Ingreso directo de estudiantes destacados de colegios seleccionados)

Admisión Selectiva

Admisión Preferente

Evaluación del talento Académico

Reconocimiento del Rendimiento escolar

Tercio Superior de colegios seleccionados
Tabla 3. Rendimiento académico universitario según cohorte.

\begin{tabular}{cccccc}
\hline Cohorte & N & $\overline{\boldsymbol{x}}$ & DS & T & p \\
\hline 2008 & 104 & 13,53 & 2,07 & & \\
2009 & 90 & 13,56 & 2,89 & & \\
& & & & & \\
\hline
\end{tabular}

$\mathrm{P} \geq 0,05$

Tabla 4. Rendimiento académico universitario según sexo.

\begin{tabular}{cccccc}
\hline Género & $\mathbf{n}$ & $\overline{\boldsymbol{x}}$ & $\mathbf{D S}$ & $\mathbf{T}$ & $\mathbf{P}$ \\
\hline Masculino & 135 & 13,76 & 2,11 & & \\
& & & & 1,81 & 0,07 \\
Femenino & 59 & 13,06 & 3,11 & & \\
\hline
\end{tabular}

Nivel de significación: 5\%

Estadístico de prueba: Prueba de t de Student lación no fue estadísticamente significativa $(\mathrm{P}>0,05)$; estos resultados concuerdan con los reportados por Girón y Guevara quienes afirman que una vez que los estudiantes ingresan a la universidad toma mayor importancia la dedicación al estudio y los procesos de enseñanza aprendizaje $(11,12)$.

Actualmente parece haber consenso en que las pruebas de admisión no son un buen predictor del rendimiento académico, y las evidencias a favor de la postura contraria no son concluyentes, en razón de que las variables que se registran al ingreso se disipan en la medida en que un alumno pasa de un curso a otro, y en su lugar pasan a ocupar indicadores del rendimiento parcial influidos por las características de la evaluación de la carrera, lo cual reorienta la atención hacia otras variables que intervienen en el rendimiento académico universitario $(6,12)$.

Cabe resaltar que la modalidad de ingreso por Factor Excelencia en la Facultad de Estomatología de la UPCH mantiene las características con las cual fue instituida, con lo cual podemos comparar los resultados del presente estudio con los reportados por Rivera en el 2004 en ingresantes en las cohortes $2001 \mathrm{y}$ 2002 de la misma facultad(13). Rivera encontró diferencias significativas entre la modalidad de ingreso por Factor Excelencia y el rendimiento académico universitario, dicho estudio difiere metodológicamente del presente, en el cálculo del rendimiento académico ya que tomó como referencia el promedio simple de las notas, mientras que la presente investigación toma el promedio ponderado de las notas obtenidas, además que la población 
Tabla 5. Diferencias del rendimiento académico universitario según modalidad de ingreso.

\begin{tabular}{lccccc}
\hline Modalidad de ingreso & $\mathbf{N}$ & $\overline{\boldsymbol{x}}$ & $\mathbf{D S}$ & $\mathbf{F}$ & $\mathbf{p}$ \\
\hline Factor Excelencia & 117 & 13,5 & 2,7 & & \\
Examen de Admisión & 43 & 13,6 & 2,0 & & \\
CEPREU & 31 & 13,6 & 2,4 & 0,03 & 0,97 \\
\hline
\end{tabular}

Nivel de significación: $5 \%$

Estadístico de prueba: Análisis de varianza

estudiada representó el 50\% del tamaño en comparación con la presente investigación (13).

Tomando como referencia la Clasificación de los Sistema de Selección de Ramallo y Sigal (5), la Facultad de Estomatología de la UPCH clasifica su Sistema de Admisión como de intensidad media, referida a la selección mediante un Examen Eliminatorio sin cupo determinado, lo cual puede afectar desfavorablemente su nivel de ingresantes, sin embargo esto más que una dificultad se convierte en un reto para la institución a fin de garantizar a la sociedad la formación de profesionales altamente calificados.

La asociación del rendimiento académico universitario según sexo mostró resultados homogéneos tanto para hombres y mujeres, lo cual coincide con Guevara y col. (12), Del Buey y Camarero (14), Porcel y col. (15), Gómez y Oviedo (16) y Rivera (13), éste último, en el año 2004 realizó un estudio en estudiantes del primer año de la Facultad de Estomatología de la UPCH.

Finalmente, los sistemas de admisión de la facultad de Estomatología Roberto Beltrán no demuestran ser un predictor efectivo del rendimiento académico universitario tal como muestran los hallazgos reportados en la presente investigación ver Tabla 6 , se sugiere su revisión en función de aspectos personales como contextuales que condicionan el aprendizaje y el trabajo docente, por tanto es necesario fortalecer la presencia de políticas educativas institucionales y normatividad lo suficientemente precisa que puedan afectar directamente sobre el rendimiento académico de los estudiantes, puesto que de ella depende que un estudiante se inserte al sistema universitario de manera eficiente con la debida orientación, lo cual finalmente se reflejará en los resultados académicos y en el subsecuente prestigio de la institución. El clima institucional y el compromiso de las autoridades y docentes finalmente reflejarán, cuanto éstas influyen sobre la calidad de sus egresados y su inserción exitosa en el mercado laboral.

Podemos concluir que en el presente estudio, no se encontró diferencias significativas según modalidad de ingreso y rendimiento académico universitario en la Facultad de Estomatología de la UPCH.

\section{Referencias Bibliográficas}

1. Donoso S, Hawes G. El sistema de selección de alumnos de las universidades chilenas. Discusión de sus fundamentos, re- sultados y perspectivas. Proposiçoes. 2002;13(2):109-26.

2. Beltrán R. Educación en Odontología. Lima: Universidad Peruana Cayetano Heredia; 1998.

3. Chadwick C. Teorías del aprendizaje. Santiago: Editorial Tecla; 1979.

4. Rodríguez S, Fita E, Torrado M. El rendimiento académico en la transición secundaria-universidad. Revista de educación. 2004; (334):391-414.

5. Ramallo M, Sigal V. Los sistemas de admisión de las Universidades en la Argentina. Documento de Trabajo. Buenos Aires: Universidad de Belgrano; 2010.

6. Sota J, Vexler I, Chávez H, Depaz Z. La Universidad en el Perú. Informe 2006. Dirección de Coordinación Universitaria, Ministerio de Educación, 2006.

7. Carrión E. Validación de características al ingreso como predictores del rendimiento académico en la carrera de medicina. Rev Cubana Educ Med Super 2002;16(1):5-18.

8. Poussaint AF, Clinical experience and minority group students. A perspective from Harvard Medical School Clin Orthop Relat Res. 1999; May (362):78-84.

9. Alterio G, Ramos I. Variables personales socioeconómicas y rendimiento académico (cohortes I y II 97 del Programa de medicina de la Universidad Centro Occidental Lisandro Alvarado de Barquisimeto-2001) Bol. Med. Postgrado. 2001;17(4):174-81.

10.García OR. Rendimiento académico en el primer año y su relación con las variables de ingreso en las escuelas de medicina. Rev. Med. Chile. 1995;123 (1):28-36. 
11. Girón L, González D. Determinantes del rendimiento académico y la deserción estudiantil, un programa de Economía de Pontificia Universidad Javeriana de Cali. Econ Gest Desarro. 2005;3:173201.

12. Guevara M, Terán I, Rodríguez Z. La admisión y el rendimiento académico en los estudios de medicina. Escuela de Medicina "Witremundo Torrealba" Universidad de Carabobo, Venezuela. Comunidad y Salud. 2011; 10(2):34-43.
13. Rivera A. Evaluación del rendimiento académico según modalidad de ingreso en estudiantes del 1er. año de la Facultad de Estomatología de la Universidad Peruana Cayetano Heredia en los años 2001-2002. Tesis de Bachiller. Lima, Perú. Facultad de Estomatología, Universidad Peruana Cayetano Heredia, 2004.

14.Del Buey F, Camarero F. Diferencias de género en los procesos de aprendizaje en universitarios. Psicothema. 2001;13(4):598-604.
15.Porcel E, Dapozo G, López M. Predicción del rendimiento académico de estudiantes del primer año de la FACENA (UNNE) en función de su caracterización socioeducativa. Revista Electrónica de Investigación educativa. 2010;12(2):1-21.

16.Gómez D, Oviedo R. Factores que influyen en el rendimiento académico del estudiante universitario. Educación y Humanidades. 2011;5(2):90-7. 